\title{
BIOMASS BURNING MEASUREMENTS IN EARLINET
}

\author{
Mariana Adam $^{1}{ }^{*}$, Doina Nicolae ${ }^{1}$, Livio Belegante ${ }^{1}$, Iwona S. Stachlewska ${ }^{2}$, Dominika Szczepanik ${ }^{2}$, \\ Maria Mylonaki ${ }^{3}$, Christiana Anna Papanikolaou ${ }^{3}$, Nikolaos Siomos ${ }^{4}$, Kalliopi A. Voudouri ${ }^{4}$, \\ Arnoud Apituley ${ }^{5}$, Luca Alados-Arboledas ${ }^{6}$, Juan Antonio Bravo-Aranda ${ }^{6}$, Aleksander Pietruczuk ${ }^{7}$, \\ Anatoli Chaikovski ${ }^{8}$, Michaël Sicard ${ }^{9,10}$, Constantino Muñoz-Porcar ${ }^{9}$, Ina Mattis ${ }^{11}$, Nikolaos \\ Papagiannopoulos $^{12}$, Lucia Mona ${ }^{12}$, Holger Baars ${ }^{13}$, Ulla Wandinger ${ }^{13}$, Daniele Bortoli ${ }^{14}$, Ivan \\ Grigorov $^{15}$, Zahary Peshev ${ }^{15}$, Bogdan Antonescu ${ }^{1}$ \\ ${ }^{I}$ National Institute of Research and Development for Optoelectronics - INOE 2000, Magurele, 077225, \\ Romania \\ ${ }^{2}$ University of Warsaw, Faculty of Physics, 02093, Poland \\ ${ }^{3}$ National Technical University of Athens, Department of Physics, Athens, 15780, Greece \\ ${ }^{4}$ Laboratory of Atmospheric Physics, Aristotle University of Thessaloniki, Thessaloniki, 54124, Greece \\ ${ }^{5}$ KNMI - Royal Netherlands Meteorological Institute, De Bilt, 3731, the Netherlands \\ ${ }^{6}$ Andalusian Institute for Earth System Research, Department of Applied Physics, University of Granada, \\ Granada, 18071, Spain \\ ${ }^{7}$ Institute of Geophysics, Polish Academy of Sciences, Warsaw, 01-452, Poland \\ ${ }^{8}$ Institute of Physics, NAS of Belarus, Minsk, 220072, Belarus \\ ${ }^{9}$ RSLab / CommSensLab, Universitat Politècnica de Catalunya, Barcelona, 08034, Spain \\ ${ }^{10}$ CTE-CRAE / IEEC, Universitat Politècnica de Catalunya, Barcelona, 08034, Spain \\ ${ }^{11}$ Deutscher Wetterdienst, Meteorologisches Observatorium Hohenpeißenberg, Hohenpeißenberg, 82383 \\ Germany \\ ${ }^{12}$ CNR-IMAA, C.da S. Loja, Tito Scalo, 85050, Italy \\ ${ }^{13}$ Leibniz Institute for Tropospheric Research, Leipzig, 04318, Germany \\ ${ }^{14}$ Earth Sciences Institute, Physics Department, University of Evora, Évora, 7000, Portugal \\ ${ }^{15}$ Institute of Electronics, Bulgarian Academy of Sciences, 1784, Sofia \\ *Email: mariana.adam@inoe.ro
}

\begin{abstract}
The biomass burning events are analyzed using the EARLINET-ACTRIS atmospheric profiling of aerosols using lidars. The period of 2008-2017 was chosen to analyze all the events assigned in the EARLINET database under Forest Fire category. A number of fourteen stations were considered. The data provided, ranged from complete data sets (backscatter, extinction and particle linear depolarization ratio) to single profiles (backscatter coefficient). A thorough quality control was performed. Smoke layers geometry was evaluated and the mean properties within each layer were computed. The Hysplit backward-trajectory technique and the FIRMS fire database were used to double check the source of each layer. Discussions were made under the following scenarios: fire events seen by two stations, long range transport from North America, and geographical clusters.
\end{abstract}

\section{INTRODUCTION}

Biomass burning (BB) represents an important source of atmospheric particles (aerosols) which influences the radiative transfer in the atmosphere with in general, a negative effect over the globe [1]. At the ground level or within PBL (Planetary Boundary Layer), strong BB episodes can cause large reduction of the visibility over various regions [2], which can affect the traffic and more importantly can cause serious health issues for humans through the low air quality [3-4]. The monitoring of the wild forest fires events occurring each year in Europe are reported since 2000 in the annual reports from European Commission [5]. Lidars can provide smoke measurements being able to deliver the boundaries of the smoke layers and the optical properties therein. Both the layer geometry and optical properties can be used for validation of the transport model output [6]. The optical properties can be also used in closure studies to model the microphysical properties of the aerosol [7]. Regarding the impact of BB aerosol on weather forecast, the study by Zhang et al. [8] suggests that the $\mathrm{BB}$ ' effect is seen for $A O D$ (aerosol 
optical depth) at 550nm larger than 1 .

Within this context, the use of ACTRISEARLINET database will bring more insights about the BB aerosol on both spatial and temporal evolution. Selection of results shown, focuses on particular events and one example of geographical clusters.

\section{METHODOLOGY}

\subsection{The EARLINET database}

The EARLINET database consists currently of 31 lidar stations covering most of Europe and one location outside Europe. A review of the EARLINET network is given by Pappalardo et al. [9]. There are several categories of submitted data file, one of them being Forest Fire (FF), which are built based on voluntary basis, as opposed to Climatology and Calipso categories. For the present study, we chose the 2008-2017 interval, as most of the data are submitted after 2008, while Hysplit model [10] is available from late 2007. We considered only the data with emission wavelength at 355, 532 and $1064 \mathrm{~nm}$. The initial data set contained $\sim 3700$ files at 20 stations.

\subsection{Data quality control}

Several criteria were implemented for data quality control (QC). Firstly, the EARLINET tools on QC were used to eliminate the $\mathrm{b}$ (backscatter) and $\mathrm{e}$ (extinction) files which did not pass the checks on technical characteristics or the optical properties values [11]. Secondly, additional data checks were performed in-house. After preliminary QC, 2300 files from 14 stations were considered.

\subsection{Evaluation Procedure}

- Firstly, an automatic algorithm was developed to estimate the boundaries of the aerosol pollution layers visible in the lidar profiles. Secondly, a manual check was performed over the automatic boundaries' retrievals. When the automatic retrievals were not accurate, the boundaries were corrected manually.

- Mean values of optical properties in the layers were computed

- NOAA Hysplit backtrajectory and fires location from NASA FIRMS [12] were used to select the pollution layers of $\mathrm{BB}$ origin, whereby quite often, several layers with different sources were recorded at once. Only the fires on a $100 \mathrm{~km}$ radius along backtrajectory and within $\pm 1 \mathrm{~h}$ of the airmass pass were selected. The backtrajectories for layers without fires found in this range were not considered in analyses.

- profiles with layers of BB origin are selected

- The selected events were discussed based on the intensive parameters (lidar ratio $L R$, backscatter Ångström exponent $B A E$, extinction Ångström exponent $E A E$, particles linear depolarization ratio $P D R)$.

- The measurements were quantified as "pure" (with single fire contributing) or "mixed" (more fires contributing)

Please note that all the criteria involved in various steps of the procedure will be discussed in detail during conference.

\section{RESULTS}

\subsection{Fire events seen at two stations}

Fig. 1 shows the BB detection at Thessaloniki ("th") and Athens ("at") stations on 29-31 May 2017. The square in the upper plot shows the common fire. The second plot shows the histogram of the number of fires' detections for the two stations. The third plot shows the common fire' coordinates versus time of occurrence. The bottom plot shows the common fire' coordinates as a function of measurement time at the two stations. Thus, the smoke from the fire occurring at $00: 00$ on $26^{\text {th }}(48.171 \mathrm{~N}, 30.622 \mathrm{E})$ arrived in "th" at $13: 36$ on $29^{\text {th }}$ at $1260 \mathrm{~m}$ altitude and in "at" at $08: 40$ on $31^{\text {st }}$ at $1742 \mathrm{~m}$ altitude. The intensive parameters (Fig. 2) were: $B A E @ 532 / 1064=0.52$ ("th") and BAE@355/532=1.69,BAE@532/1064 $=1.32$ ("at"). Smoke was considered "pure" (i.e. with one source) for both stations. Figures 3-4 depict backtrajectories and the fires' location for "th" and "at". As better seen on bottom plots, for each station there was only one fire contributing to the measurement. The smoke was observed after $85 \mathrm{~h} 30 \mathrm{~m}$ in "th" and $128 \mathrm{~h} 40 \mathrm{~m}$ in "at" from the time of occurrence. The common variable was BAE@532/1064. The value in "th" was smaller than the value in "at". The difference can be explained by the slightly different path and travel time. 


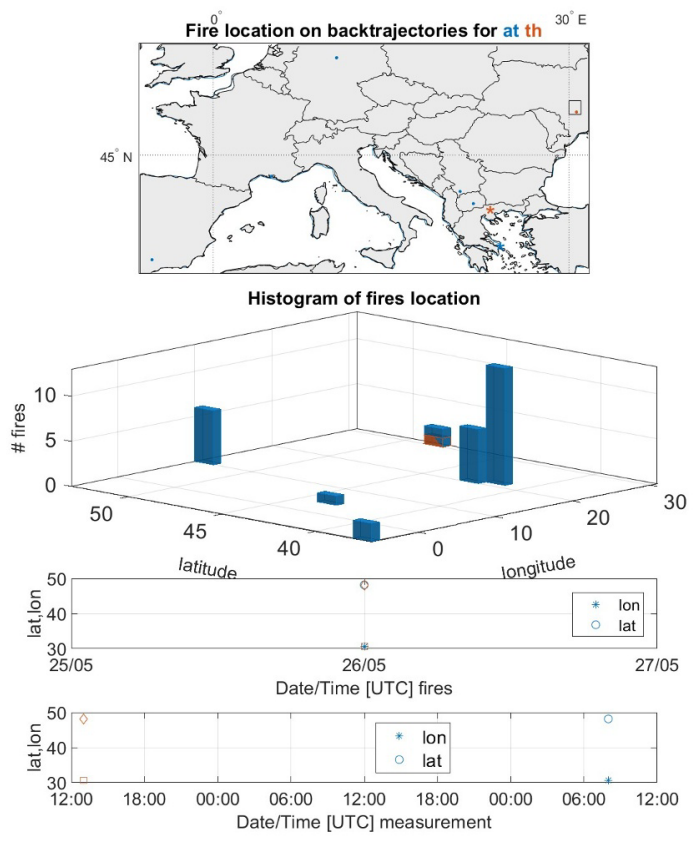

Fig. 1. Upper: location of the fires captured by "at" and "th" stations on 29 May - 2 June 2017. Square shows the common fire. Second: histogram of the number of fires' detection. Third: common fire' coordinates and time of fires' observation. Bottom: fire' coordinates versus lidar measurement time.
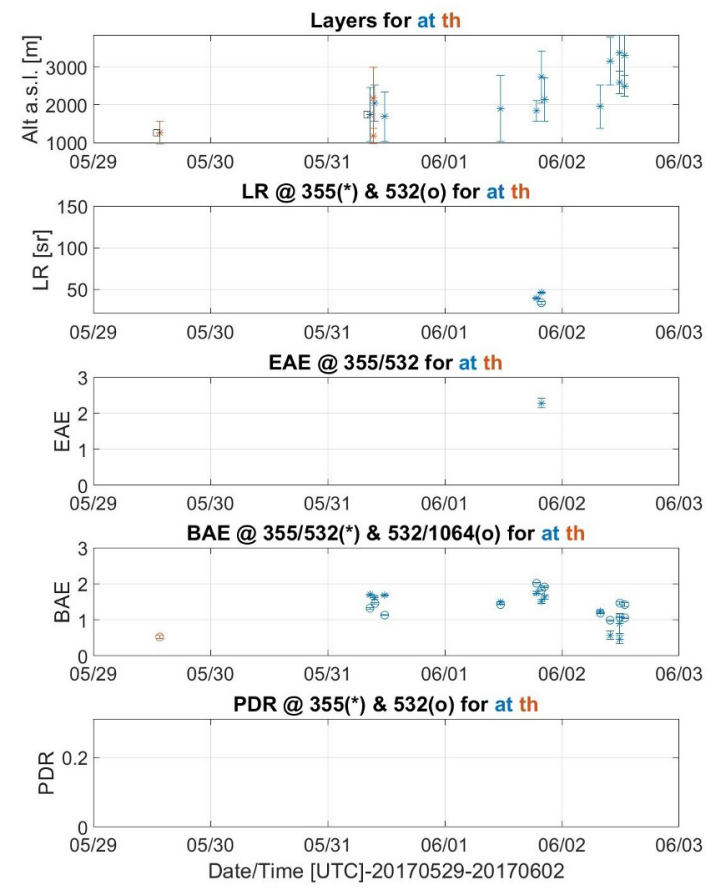

Fig. 2. Intensive variables for "at" and "th", event 29 May - 2 June 2017. Squares show the layers with BB origin.

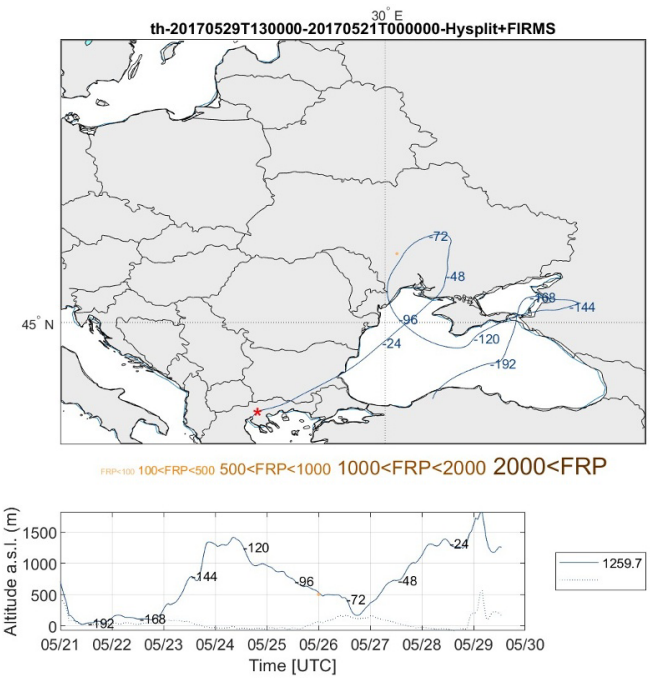

Fig. 3. Hysplit backtrajectory and fires' location along for "th" station. Backtrajectory starting time: 13:00 20170529, altitude $1260 \mathrm{~m}$ asl.
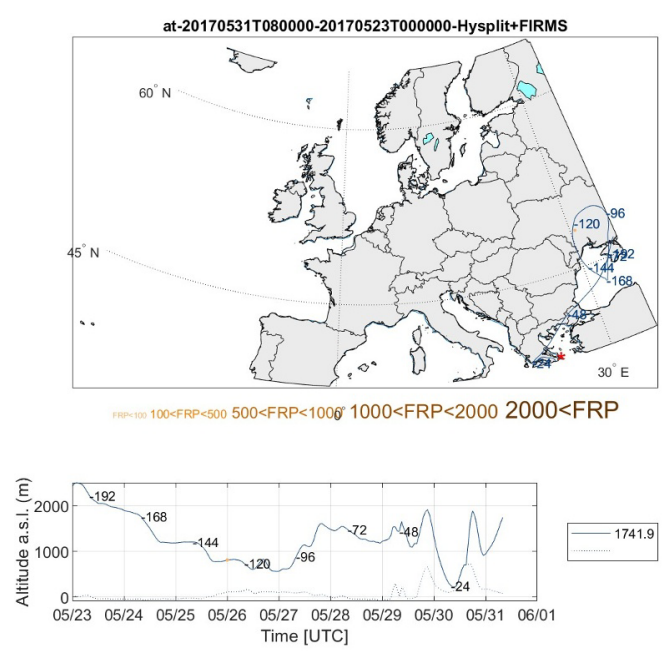

Fig. 4. Hysplit backtrajectory and fires' location along for "at" station. Backtrajectory sarting time: 08:00 20170531 , altitude $2123 \mathrm{~m}$ and $1742 \mathrm{~m}$ asl.

\subsection{Long range transport from $\mathrm{N}$ America}

We identified a number of $24 \mathrm{BB}$ events with long range transport (LRT) from $\mathrm{N}$ America. The strongest event was recorded by three stations (Belsk -be, Cabauw-ca and Warsaw -wa) during 8-10 July 2013 with the main fires occurring between 30 June to 7 July. Some of those measurements were discussed in literature (e.g. [13-14]). This event will be discussed from a larger perspective during the conference. The statistics of the intensive parameters of LRT from 
$\mathrm{N}$ America (over all 24 events) is shown in Table 1. We divided the events into two categories: "pure N America" (NA only) and "mixed" (N America + European). The values from literature on N America LRT (Lit res) were added for comparison. Note that we do not have information if the LRT values reported were pure or mixed fires (in the way we defined them). Values for "pure" cases are closer to literature except PDR.

Table 1. Mean intensive parameters for long range transport from N America.

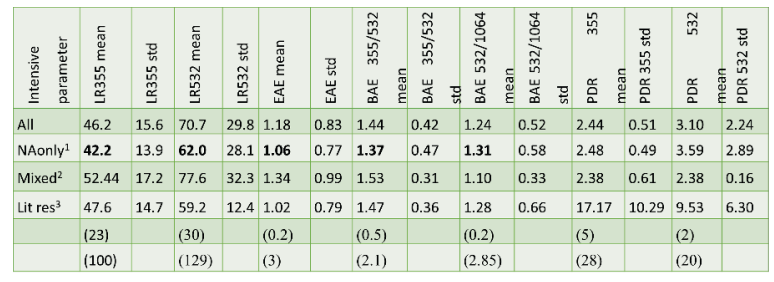

* In parenthesis, minimum and maximum values.

\subsection{Geographical clusters}

The EARLINET stations considered in BB study were split into four geographical clusters: SE Europe (Athens-at, Bucharest-bu, Potenza-po, Sofia-sf and Thessaloniki-th), SW Europe (Barcelona-ba, Evora-ev, Granada-gr), NE Europe (Belsk-be, Minsk-mi and Warsaw-wa), Central Europe (Cabauw-ca, Leipzig-le, Observatory Hohenpeissenberg-oh).
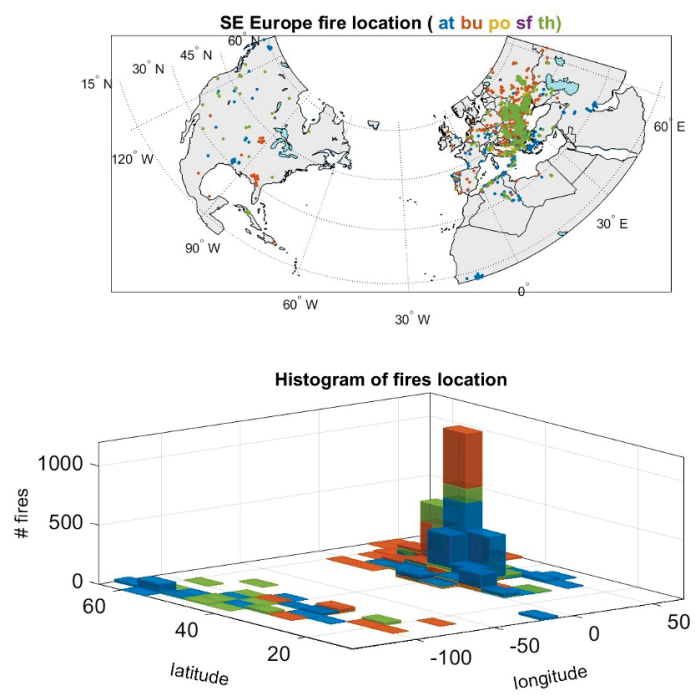

Fig. 5. SE Europe cluster (Athens, Bucharest, Potenza, Sofia, Thessaloniki). Upper plot represents the location of the fires. Lower plot represents the histogram of the number of fires' detection.
As it will be shown during the conference, each of those clusters measures predominantly smoke from fires occurring in a specific region. As an example, SE Europe cluster is shown in Fig. 5. The stations from this region record BB events with the major source in SE Europe. Each cluster will be discussed in connection with the main meteorological features of each region.

\section{CONCLUSIONS}

The Forest Fire category of the EARLINET ACTRIS database allows to perform a variety of studies on biomass burning aerosols. One can exploit fires' smoke as i) recorded by several stations, ii) long range transport (e.g. N America or W Asia), on the basis of iii) statistics on geographical clusters, and iv) the cases with complicated meteorology when one station can measure mixed BB aerosols, from different sources.

\section{ACKNOWLEDGEMENTS}

This work was supported by the H2020 ACTRIS2 No 654109 and the Romanian National contracts 18N/08.02.2019 and 19PFE/17.10.2018.

\section{REFERENCES}

[1] IPCC, https://www.ipcc.ch/ipccreports/tar/wg1/235.htm (2001) [2] M. Adam, et al. J. Geophys. Res., 109, D16S02, doi:10.1029/2003JD004047 (2004)

[3] A. Sapkota, et al. Environ. Sci. Technol., 39, 24-32 (2005)

[4] E. Alonso-Blanco, et al. Atmos. Chem. Phys., 18, 10615-10643 (2018)

[5] http://effis.jrc.ec.europa.eu/reports-andpublications/annual-fire-reports/ (2000-2017)

[6] B. L. van Drooge, et al. Atmos. Poll. Res., 7, 494502 (2016)

[7] M. Fiebig, et al. Atmos. Chem. Phys., 3, 881-891 (2003)

[8] J. Zhang, et al. Atmos. Chem. Phys., 16, 6475-6494 (2016)

[9] G. Pappalardo, et al. Atmos. Meas. Tech., 7, 23892409, 2014

[10] A. F. Stein, et al. Bull. Amer. Meteor. Soc., 96, 2059-2077 (2015)

[11] https://www.earlinet.org/index.php?id=125

[12] https://firms.modaps.eosdis.nasa.gov/

[13] L. Janicka, et al. Atmos. Environ., 169, 162-174

(2017)

[14] P. Ortiz-Amezcua, et al. Atmos. Chem. Phys., 17, 5931-5946 (2017) 